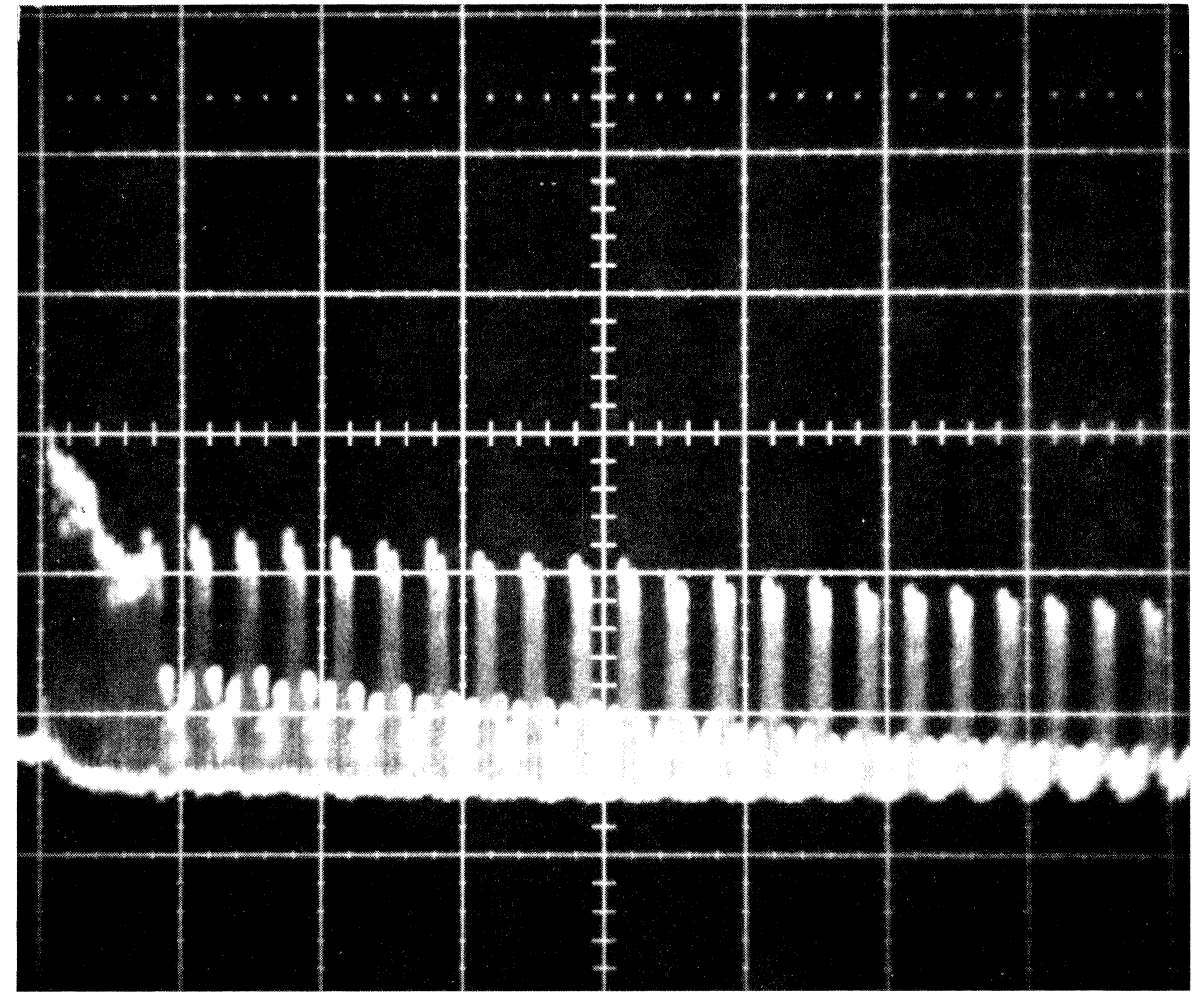

be taken by octupoles required to cope with the 'resistive wall instability'. (The effect of the octupoles on ejection conditions has not yet been worked out.) Disturbance of the beam at transition seems to be no problem. Instabilities due to the presence of electrons from the ionization of the residual gas could be troublesome but the introduction of a gap in the accelerated beam would cure it. Beam-component interactions which could perturb the beam do not seem to be a worry with the possible exception of interactions with the r.f. cavity structure.

A look was also taken at possible future storage ring schemes, the aim being simply to keep options open to avoid doing anything unnecessarily which would make the SPS accelerated beams less suitable for storage rings should this seem an appropriate development many years from now. Among the schemes examined were a superconducting conversion of the ISR for higher energies (where the SPS characteristics look good), a by-pass scheme (where potential luminosities and costs do not look encouraging) and electron-proton schemes (where physics interest is currently high).

Another study is planned for the autumn when the work will concentrate on beam diagnostic equipment for the SPS.

\section{Leptonic decays of hyperons}

An experiment to study the leptonic decays of negative hyperons is under way in the East Hall of the $28 \mathrm{GeV}$ proton synchrotron. This is another example of an experiment which was impossible prior to the recent setting up of hyperon beams at Brookhaven and at CERN.

The experiment is being carried out by a collaboration of scientists from CERN, Ecole Polytechnique and Orsay.
The same team is now publishing the results of the previous experiment on the hyperon beam in which they looked at the sigma minus-proton total crosssection where the quark model predicts a relationship between this crosssection and other known cross-sections (the beam-line and the experiment were described in vol. 11, page 191). Expressing their result briefly the quark model has stood up to the test.

The new experiment adds two streamer chambers to the collection of detectors and the sequence of equipment now reads as follows: the high energy slow ejected beam from the PS hits a target to produce the hyperons (higher energy increasing their lifetime) which are filtered out through a focusing and analysing system crowded together in only $3.7 \mathrm{~m}$ of beamline to preserve as many of the shortlived hyperons as possible. The beamline consists of two bending magnets and two short superconducting quadrupoles. A DISC (differential Cherenkov counter) at the output of the beam-line selects between the different particles. A useful flux of about 100 negative sigma hyperons is obtained from a beam of $2 \times 10^{11}$ protons on the target. Multiwire proportional chambers on either side of the DISC give the direction of an incoming hyperon to a precision of $\pm 0.5 \mathrm{mrad}$.

A small sweeping magnet gets rid of low energy electrons just before the particles enter the first streamer chamber. This chamber is $3 \mathrm{~m}$ long, built by Orsay and Ecole Polytechnique, and is performing well. The leptonic decays of interest (such as negative sigma hyperon decaying into neutron, electron and antineutrino or into lambda, electron and antineutrino) take place in the streamer chamber and the charged particle tracks can be photographed. A threshold Cherenkov counter follows to identify the electrons (pions, which occur much more 


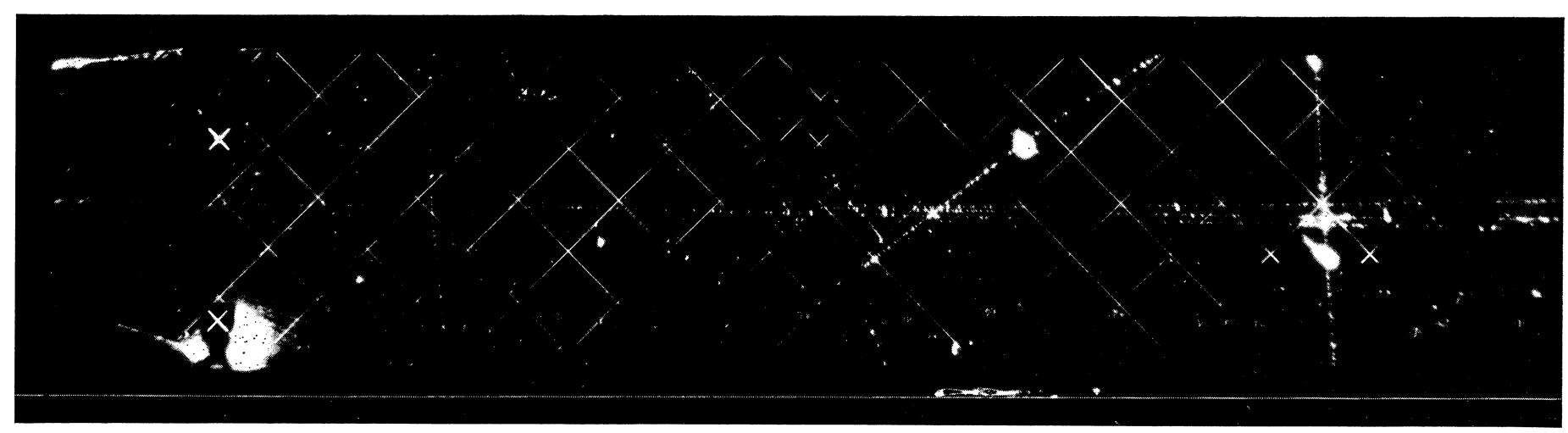

frequently in sigma decays, do not give a signal) and there is then a second streamer chamber in a $0.8 \mathrm{~T}$ magnetic field $1.4 \mathrm{~m}$ long. This detector is used to measure the momenta of the electron and the charged decay products of the lambda. Proton counters give further handles on the lambda decay products and a neutron detector, consisting of optical spark chambers interleaved with thick iron plates, catches the neutron from the decay.

This battery of detectors ensures that the required information is collected and avoids,. as far as possible, collecting information about the many other types of event which, with a smaller array of detectors, could be mistaken for the events which are to be studied.

The physics interest lies in obtaining coupling constants for axial and vector currents with greater precision than has been achieved previously. It is predicted that only the axial vector current is involved in the sigma leptonic decay to a lambda. Also it should be possible, with the accuracy which the experiment hopes to achieve, to see the breakdown of the Cabibbo model of weak interactions. The model takes SU3 as an exact symmetry whereas in fact it should be broken within about $10 \%$ limits. The experiment could register the effect of the symmetry breaking.

An experiment on sigma minus decays is also gathering data with the hyperon beam at Brookhaven. The experiment is carried out by a Brookhaven, NAL, Yale collaboration.

Another interesting demonstration that yesterday's hypothesis can become today's commonplace, is the fact that the Brookhaven hyperon beam is yielding on observable omega minus hyperon at the rate of about one per hour. Less than ten years ago the omega minus was just a gleam in theoretical eyes and prior to the hyperon beams only 30 of them had ever been identified.

\section{Minnie}

In the last issue we carried a piece on 'Bessy' a scanning and preliminary measuring machine specifically designed to cope with film from the $3.7 \mathrm{~m}$ European bubble chamber, BEBC. That device has proved extremely popular and has been ordered by many European research centres. Another machine, which like Bessy has been designed with economy very much in mind and with film from a particular chamber in view, is one called 'Minnie'.

'Minnie' is used for scanning and for measurement in the image plane of film from the heavy liquid bubble chamber Gargamelle. The efficient analysis of experiments in Gargamelle requires apparatus with rather complicated features in general but a good portion of the analysis can be done without them. 'Minnie' supplements the existing apparatus when these features are not required. It was designed and built at CERN very rapidly (about six months having elapsed between the first tests and the appearance, in September 1971, of the prototype which has been in uninterrupted production since then).

The machine could be manufactured in the workshops of most physics laboratories. The prototype without the measurement device cost about 10000 Swiss francs for materials and about 800 hours work. At the moment, twelve are planned or are being built in various European research centres.

Many of the simplifications used in 'Minnie' are the same as those used by 'Bessy' (see last issue page 127). But it was also necessary to find a simple way of handling the complicated film format of Gargamelle which takes eight views spread out on two films for each photo. Views of the same photo are separated by sixteen other views of other photos. Instead of each film undulating like a Loch Ness monster between separate film gates it is wound in a spiral so that the views of the same photo are adjacent and all 8 can be held in a single film gate $675 \mathrm{~mm}$ long and $70 \mathrm{~mm}$ wide. The eight lenses are also on a single plate.

With a magnification of $12 \times$ this compact arrangement makes it possible to hang the projector from the roof in a room of normal height without 